\title{
Remission bei Depression erreichen
}

D epressive Störungen gehören heute zu den volkswirtschaftlich bedeutsamsten Erkrankungen, und nach den Schätzungen der Weltgesundheitsorganisation (WHO) wird ihre Bedeutung in den nächsten Jahrzehnten weiter zunehmen. Nach der „Global Burden of Disease“-Studie der WHO gehören depressive Störungen schon jetzt zu den Erkrankungen, die zum größten Verlust an Lebensjahren aufgrund der Beeinträchtigung durch die Störung führen. Depressionen werden von den Betroffenen als beeinträchtigender erlebt als die meisten somatischen Erkrankungen. Hinzu kommt, dass viele chronische somatische Erkrankungen im Verlauf durch das Hinzutreten einer depressiven Störung weiter verkompliziert werden. Bei vielen Erkrankten wird die Diagnose nur mit großer Verzögerung oder gar nicht gestellt, und wenn sie richtig gestellt wird, erfolgt die Behandlung oft nur unzureichend. Wahrscheinlich werden weniger als $10 \%$ der Betroffenen medikamentös adäquat behandelt. Dies liegt auch daran, dass nach wie vor nicht nur z.B. Patienten mit schizophrenen Störungen oder Substanzabhängigkeiten von gesellschaftlicher Stigmatisierung betroffen sind, sondern auch Patienten mit depressiven Störungen. Auch dies trägt dazu bei, dass viele von dieser schwerwiegenden Erkrankung Betroffene den Gang zum Arzt scheuen. Konsens ist heute auch, dass das Ziel der Behandlung nicht mehr nur die Response, d.h. das (partielle) Ansprechen auf die Therapie, sein darf, sondern dass in jedem Fall die Remission angestrebt werden muss.

Wenn wir also getreu dem Leitthema dieses Heftes bei möglichst vielen betroffenen Patienten die Remission der Erkrankung erreichen wollen, so müssen unsere Bestrebungen heute zwei wesentliche Zielrichtungen haben: Zunächst müssen wir den Kreis derjenigen Patienten, die wir überhaupt mit unseren therapeutischen Maßnahmen erreichen, erheblich vergrößern. Wenn die Diagnose gestellt und die Behandlungsnotwendigkeit erkannt wurde, müssen alle uns heute zur Verfügung stehenden Maßnahmen ausgeschöpft werden, um eine Vollremission der Erkrankung zu erreichen. In diesem Heft beschreibt Frau Christine Norra, jetzt Göttingen, wie sie in der Region Aachen unter der Federführung von Frank Schneider am dortigen Universitätsklinikum nach dem Muster des im BMBF-geförderten „Kompetenznetz Depression/Suizidalität“ prototypisch aufgebauten „Bündnis gegen Depression“ ein ähnliches Aktionsprogramm in einer mittleren Großstadt aufgelegt hat, um die Versorgung depressiv Erkrankter zu verbessern und ihre Stigmatisierung zu vermindern. Martin Hautzinger, Tübingen, betont in seinem Beitrag, wie sinnvoll gerade bei leichten bis mittelschweren Depressionen auch (kognitiv) verhaltenstherapeutische Maßnahmen sind, ggf. in Kombination mit einer Pharmakotherapie, um auch langfristig stabile Remissionen zu erreichen. In vielen Fällen - und dazu zählen nicht nur Patienten mit schweren oder psychotischen Depressionen - wird jedoch nur eine suffiziente Therapie mit Antidepressiva eine vollständige Remission erlauben. Michael Bauer und Mitarbeiter, Berlin, geben in ihrem Beitrag eine Übersicht über die vielfältigen Möglichkeiten der modernen Pharmakotherapie. Dabei stellen sie die Bedeutung systematisierter Therapiealgorithmen heraus, um möglichst hohe Remissionsraten zu erreichen.

Die Autoren dieses Heftes zeigen, dass sich die Versorgungs- und Behandlungsmöglichkeiten depressiver Störungen heute erheblich verbessert haben. Die gemeinsame Anstrengung vielfältiger Berufsgruppen ist nötig, um auf diesem Weg weiter voranzukommen.

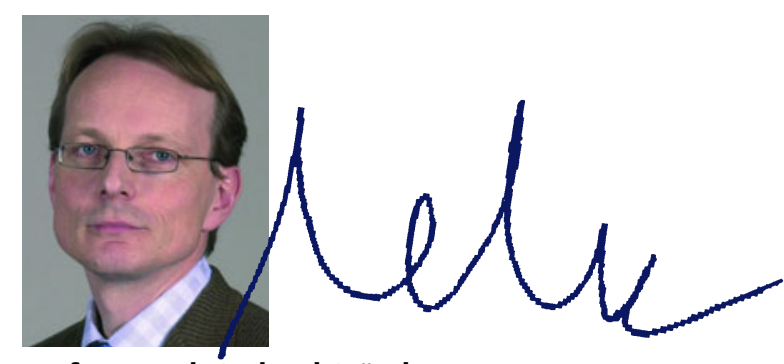

Prof. Dr. med. Gerhard Gründer, Aachen 